\title{
Evaluation of prophylactic antibiotic administration at the surgical ward of a major referral hospital, Islamic Republic of Iran
}

\author{
G. Vessal, ${ }^{1}$ S. Namazi, M.A. Davarpanah ${ }^{2}$ and F. Foroughinia ${ }^{7}$
}

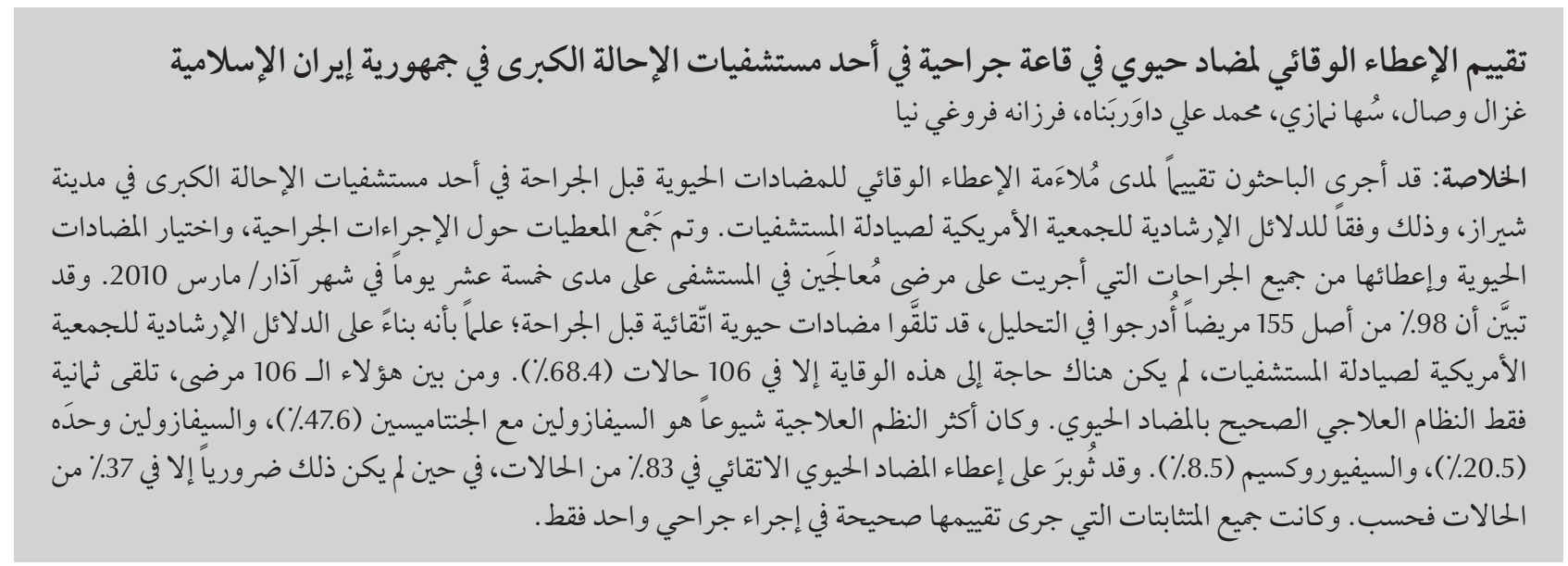

ABSTRACT We evaluated the appropriateness of antibiotic prophylaxis administered before surgery at a major referral hospital in Shiraz, against the American Society of Hospital Pharmacists (ASHP) guidelines. Data on surgical procedure, choice of antibiotic and administration were gathered for all surgeries performed on hospitalized patients during 15 days in March 2010. Of 155 patients included in the analysis, 98\% received prophylactic antibiotic before surgery; according to ASHP guidelines, prophylaxis was needed in only 106 (68.4\%). Of these 106, only 8 patients received the correct antibiotic regimen. The commonest regimens administered were cefazolin + gentamicin (47.6\%), cefazolin (20.5\%) and cefuroxime (8.5\%). Antibiotic prophylaxis was continued in $83 \%$ of cases, while this was necessary in only $37 \%$. In only 1 surgical procedure were all evaluated parameters correct.

Évaluation de l'administration prophylactique d'antibiotiques au service de chirurgie d'un grand hôpital de recours en République islamique d'Iran

RÉSUMÉ Nous avons évalué l'opportunité de l'administration d'antibiotiques prophylactiques avant une intervention chirurgicale dans un grand hôpital de recours à Chiraz, conformément aux directives de l'American Society of Health-System Pharmacists [Société américaine des pharmaciens du système de soins de santé]. Les données sur l'acte chirurgical, le choix des antibiotiques et leur administration ont été collectées pour toutes les interventions chirurgicales réalisées chez des patients hospitalisés pendant 15 jours en mars 2010. Sur 155 patients, 98 \% d'entre eux ont reçu une antibiothérapie prophylactique préopératoire ; selon les directives de I'American Society of Health-System Pharmacists, la prophylaxie se justifiait chez seulement 106 patients (soit 68,4 \% d'entre eux). Parmi ces 106 cas, seulement huit ont reçu un traitement antibiotique adapté. La céfazoline associée à la gentamicine $(47,6 \%)$, la céfazoline (20,5\%) et la céfuroxime (8,5\%) étaient les traitements les plus fréquemment administrés. La prophylaxie antibiotique a été poursuivie dans $83 \%$ des cas, alors que cela ne s'avérait nécessaire que pour 37 \% d'entre eux. Une seule intervention chirurgicale réunissait tous les paramètres corrects, selon notre évaluation.

'Department of Clinical Pharmacy, Faculty of Pharmacy, Shiraz University of Medical Sciences, Shiraz, Islamic Republic of Iran (Correspondence to G.Vessal:gvessal@yahoo.com; gvessal@sums.ac.ir).

${ }^{2}$ Department of Internal Medicine, Nemazi Hospital and Shiraz HIV/AIDS Research Center, Shiraz University of Medical Sciences, Shiraz, Islamic Republic of Iran.

Received: 22/11/09; accepted: 11/01/10 


\section{Introduction}

Antibiotic prophylaxis has been routinely used for more than 25 years to prevent postoperative infectious complications [1]. Appropriately administered antibiotic prophylaxis before surgery reduces the incidence of surgical site infection. However, inappropriate prescribing and excessive use of antimicrobials increase antibiotic resistance as well as adverse drug events and costs [2]. Multidisciplinary development of evidence-based prophylaxis guidelines incorporating local microbiology and resistance patterns can improve antimicrobial use [3]. Very often surgeons do not comply with short courses of prophylactic antibiotics before surgery or they use broad-spectrum antibiotics [2].

Increasing health-care costs have led hospitals to review procedures to adjust their budgets. At the same time, concerns about antimicrobial resistance have pressured infection control specialists to decrease antimicrobial usage. In order to develop a guideline for surgical prophylaxis at our hospital, a major referral hospital in the south of the Islamic Republic of Iran, we collected data on the performance of our current perioperative practices for antimicrobial prophylaxis. Antibiotic prophylactic regimens administered before surgical procedures were evaluated and compared with guidelines of the American Society of Hospital Pharmacists (ASHP), which are the most complete guidelines available and internationally recognized [4].

\section{Methods}

A data collection form was designed by a clinical pharmacist, and approved by the Pharmacy \& Therapeutics Committee of the Nemazi Hospital in Shiraz. The form included items regarding patient demographics, type of surgical procedure, drug history, drug allergy, choice of antibiotic regimen, dose, time of administration, and number of doses (pre-, intra-, and post-operatively). A panel of experts assessed whether the data collection form measured what it was intended to measure, and if it was comprehensive enough to collect all the information needed to address the purpose and goals of the study. We then did a pilot test for 20 patients and made appropriate changes based on expert opinion.

The form was filled out prospectively by 6 pharmacists interns for all surgical procedures performed during a 15-day period (March 2008) on hospitalized patients. Retrospective follow-up was performed by 2 clinical pharmacists and an infectious diseases specialist, to the day of discharge, using patient files.

To avoid difficulties in distinguishing prolonged prophylaxis from postoperative infection treatment, surgeries that were in the contaminated or dirty category as well as those patients who received therapeutic antibiotic before surgery or those with signs and symptoms of infection after surgery were excluded from the study. These are the categories that need therapeutic antibiotic administration as per ASHP guidelines. The contaminated category included: any penetrated trauma $(<4$ hours old), major technique break or major spillage from the gastrointestinal tract and any acute non-purulentinflammation; the "dirty" category included: any penetrated trauma ( $>4$ hours old), purulent inflammation or abscess (active infectious process) or preoperative perforation of viscera.

The compliance of prophylactic antibiotic administration was assessed against the published guidelines of ASHP [4]. The following 6 aspects of antimicrobial prophylaxis were assessed:

- indication: appropriate decisionmaking regarding use or non-use of antimicrobial prophylaxis,
- choice: antibiotic choice for patients with and without allergy,

- dose,

- timing of dose: at a fixed time before incision (within 60 minutes prior to skin incision),

- repeated dosing during procedure,

- duration of use.

Extra costs due to incorrect or overuse of antibiotics were calculated as: cost of incorrect usage -cost of correct usage. The cost of incorrect antibiotic(s) use was calculated as: cost of a single vial $x$ number of vials administered incorrectly, while the cost of correct antibiotic use as: cost of a single vial $\times$ correct number of vials needed.

All data were analysed using SPSS, version 12 . Frequencies and percentages were calculated.

\section{Results}

A total of 166 surgeries performed on hospitalized patients were evaluated during the 15-day period; $92.8 \%$ were elective while $7.2 \%$ were emergency operations. Just over $60 \%$ (101) of the patients were male. The mean age and standard deviation (SD) of the patients was 39.2 (SD 22.6) years (range 0-84 years).

Cardiothoracic, gastrointestinal, genitourinary, neurosurgical and orthopaedic procedures were the most frequent surgeries performed, accounting for $18.7 \%, 17.5 \%, 16.9 \%, 13.9 \%$, and $11.4 \%$ of the surgeries respectively (Table 1).

Patients who received therapeutic antibiotic $(n=11)$ before surgery, were excludedfromtheanalysis. Thisincluded operations classified as "contaminated" or "dirty". Therefore further analysis was performed on 155 operations.

Table 2 shows that in the majority of the surgeries included in the analysis [106 (68.4\%)], the prophylactic antibiotics administered were necessary according to the ASHP guidelines. 


\begin{tabular}{lcc}
\hline $\begin{array}{l}\text { Table 1 Type of surgeries performed during } \\
\text { Shiraz, Islamic Republic of Iran }(\boldsymbol{n}=\mathbf{1 6 6})\end{array}$ & -day period in Nemazi Hospital, \\
\hline Type of surgery & 31 & $\%$ \\
\hline Cardiothoracic & 29 & 18.7 \\
Gastrointestinal & 28 & 17.5 \\
Genitourinary & 23 & 16.9 \\
Neurosurgical & 19 & 13.9 \\
Orthopaedic & 9 & 11.4 \\
Head \& neck & 8 & 5.4 \\
Vascular & 1 & 4.8 \\
Obstetric/gynaecologic & 18 & 0.6 \\
Others $^{\mathrm{a}}$ & & 10.8 \\
\hline
\end{tabular}

aInguinal hernia, umbilical hernia, tissue expansion, skin graft, excision of scar tissue, portal catheter, adrenalectomy, chest wall mass, herniorrhaphy, tissue expander removal and splenectomy.

However, in almost $30 \%$ of the surgeries, a prophylactic antibiotic was given, although it was not required according to the guideline.

The prophylactic antibiotics administered in 152 patients are shown in Table 3. A combination of cefazolin and gentamicin was administered preoperatively in $93.5 \%$ of the cardiothoracic procedures, $70.0 \%$ of the genitourinary, $95.5 \%$ of the neurosurgical, and $53.3 \%$ of orthopaedic procedures.

The choice of antibiotic was consistent with ASHP guidelines in 8 (7.5\%) procedures that required prophylactic antibiotics. Regardless of the choice of antibiotic, timing was correct in $76 \%$ of the 106 operations. In 4 operations the antibiotic was repeated during the procedure, but only 1 required a repeat dose according to the ASHP guidelines. Prophylactic antibiotic was continued in 88 patients after their surgery; however continuation was needed in only 40 of these patients.
Table 4 shows the frequency of correct prophylactic antibiotic administration in the 106 operations that required a prophylactic antibiotic.

The average extra cost per patient due to misuse of antibiotics was 92528 (SD 133 650) rials, which is approximately equal to US\$ 9. The total extra cost due to misuse of antibiotics during the 15-day period was 15267170 rials (US\$ 1471).

\section{Discussion}

The present study demonstrates that, disappointingly, adherence to the ASHP guidelines for antimicrobial prophylaxis in our hospital is far from optimal. Only $0.9 \%$ of surgical procedures adhered to all antibiotic prophylaxis guideline parameters. These results are consistent with those of similar studies in Canada, Nicaragua, Islamic Republic of Iran and Jordan, where rates of complete adherence to practice guidelines

\begin{tabular}{lcc}
\hline $\begin{array}{l}\text { Table 2 Evaluation of prophylactic antibiotic indication before surgery in Nemazi } \\
\text { Hospital, Shiraz, Islamic Republic of Iran }\left(\boldsymbol{n}=\mathbf{1 5 5}^{\mathbf{a}}\right)\end{array}$ & \multicolumn{1}{c}{} \\
\hline Prophylactic antibiotic: & 106 & 68.4 \\
Required and administered & 3 & 1.9 \\
Not required and not administered & 0 & 0.0 \\
Required but not administered & 46 & 29.7 \\
Not required but administered &
\end{tabular}

a Patients who received therapeutic antibiotic $(n=11)$ before surgery were excluded from the analysis. were $5 \%[5], 0.7 \%$ [6], 0.3\% [7], and zero [8] respectively. Other studies however have reported higher rates of adherence to antimicrobial prophylaxis guidelines. Gorecki et al. (United States of America), van Kasteren et al. (the Netherlands), Lallemand et al. (France), and Voit et al. (United States of America) reported adherence rates of $26 \%, 28 \%, 41 \%$ and approximately $50 \%$ in their studies [9-12]. It is worth mentioning that adherence in all of the previously mentioned studies, except the ones performed by Al-Momaney et al. (Jordan) [8] and Askarian et al. (Iran) [7] was compared with local rather than international guidelines. The higher adherence in studies that used local guidelines $(7 \%-50 \%)$ suggests that adherence to these guidelines may be easier to achieve than adherence to international guidelines.

Appropriate decision-making regarding use or non-use of prophylactic antibiotics, choice of antibiotic and duration of prophylactic antibiotic use were 3 parameters with the least adherence to the standard guidelines in the present study. Prophylactic antibiotics were administered in $98 \%$ of the procedures, while only $68 \%$ of the surgeries required them according to the guidelines. This suggests that surgeons in our country are aware of the value of antibiotics in preventing surgical site infections, but as in some other countries $[13,14]$, overuse of antibiotics is common. In contrast, under-use has been reported in a study from a developed country [15].

The choice of antibiotic complied with guidelines in only $7.5 \%$ of the surgical procedures in this study. This low rate is disappointing, particularly as selection of the appropriate antibiotic for prophylaxis was much higher in the United States of America (95\% appropriate) [15] and Brazil (75\%) [16]. However, it is similar to India (12\%) [17] and higher than reported in Jordan (1.7\% of the procedures) [8]. The high rate of inappropriate choice of 


\begin{tabular}{|c|c|c|}
\hline Type of surgery & $\begin{array}{l}\text { Antibiotic regimen } \\
\text { administered }\end{array}$ & No. \\
\hline \multirow[t]{3}{*}{ Cardiothoracic } & Cefazolin + gentamicin & 29 \\
\hline & Cefuroxime & 1 \\
\hline & Ceftriaxone + clindamycin & 1 \\
\hline \multirow[t]{8}{*}{ Gastrointestinal } & Ceftriaxone + metronidazole & 6 \\
\hline & Cefuroxime & 6 \\
\hline & Cefazolin & 4 \\
\hline & Ceftriaxone & 3 \\
\hline & Ceftizoxime + metronidazole & 2 \\
\hline & Cefuroxime + cefazolin & 1 \\
\hline & Ampicilline + gentamicin & 1 \\
\hline & Metronidazole & 1 \\
\hline \multirow[t]{4}{*}{ Head \& neck } & Cefuroxime & 4 \\
\hline & Cefazolin & 3 \\
\hline & Ampicilline + gentamicin & 1 \\
\hline & Ceftizoxime & 1 \\
\hline Obstetric/gynaecologic & Cefazolin & 1 \\
\hline \multirow[t]{5}{*}{ Orthopaedic } & Cefazolin + gentamicin & 8 \\
\hline & Cefazolin & 3 \\
\hline & Cefuroxime + cefazolin & 2 \\
\hline & Ceftriaxone & 1 \\
\hline & Ampicillin + gentamicin & 1 \\
\hline \multirow[t]{4}{*}{ Vascular } & Cefazolin & 3 \\
\hline & Cefazolin + gentamicin & 2 \\
\hline & Ceftriaxone & 1 \\
\hline & Cefuroxime & 1 \\
\hline \multirow[t]{3}{*}{ Genitourinary } & Cefazolin + gentamicin & 19 \\
\hline & Cefazolin & 8 \\
\hline & Ampicillin + gentamicin & 1 \\
\hline \multirow[t]{2}{*}{ Neurosurgery } & Cefazolin + gentamicin & 21 \\
\hline & Ampicillin + gentamicin & 1 \\
\hline \multirow[t]{3}{*}{ Other } & Cefazolin & 12 \\
\hline & Cefuroxime & 2 \\
\hline & Ceftizoxime + metronidazole & 1 \\
\hline
\end{tabular}

prophylactic antibiotic and unnecessary continuation in our study may be due to the unavailability of a unit-based clinical pharmacist to assist physicians in correct choice of medications according to guidelines. Another reason may be because medical residents are not as fully trained on medication choices as they are on diagnosing diseases and performing procedures. Our study was performed in a university teaching hospital, and medical faculty members
The ASHP recommends prophylaxis with cefazolin as a single agent for most procedures. However, in the present study a combination of aminoglycoside with cefazolin was the most common regimen used, while the use of third generation cephalosporins were also not uncommon. Third generation cephalosporins, aminoglycosides and fluoroquinolones should not be used for surgical site infection prophylaxis because of less activity against staphylococci infections compared to cefazolin, emergence of resistance, and high cost $[18,19]$. The combination of cefazolin and gentamicin was used most commonly in cardiothoracic, genitourinary and neurosurgical operations. This antibiotic combination may be used in situations when certain Gram-negative bacteria not responsive to cefazolin are involved in addition to Gram-positive bacteria such as staphylococci. However, Gram-negative bacteria involved in the above surgeries are mostly enteric Gram-negative bacilli [20] and cefazolin alone can cover these pathogens [21]; thus there is no need for this combination.

In the present study antibiotics were continued in 88 of the procedures that required prophylactic antibiotics, however continuation was not necessary in $55 \%$ of these surgeries. Similarly in the study performed by Al-Momany et al. in Jordan [8], the duration of antibiotic prophylaxis was longer than recommended in $58.9 \%$ of patients. This has been reported by other researchers $[7,14,17,22]$. According to international guidelines, a single dose of antibiotic is enough for most surgical procedures. Prolonged antibiotic prophylaxis is not only of no benefit but also potentially harmful to patients due to toxicity, risk of super-infection and the risk of inducing more bacterial resistance $[16,23]$.

The timing of administration of prophylactic antibiotics is important and this was correct in $76.5 \%$ (81 of 106 that required prophylaxis) of the surgeries performed. This is similar to the studies 


\begin{tabular}{lcc}
\hline Table 4 Antibiotic administration in surgeries that required prophylaxis $(\boldsymbol{n}=\mathbf{1 0 6})$ \\
\hline Antibiotic administration & No. & $\%$ \\
Correct choice of antibiotic & 8 & 7.5 \\
Correct choice + correct dose & 8 & 7.5 \\
Correct choice + correct dose + correct time & 2 & 1.9 \\
Correct choice + correct dose + correct continuation & 1 & 0.9 \\
Correct choice + correct dose + correct time + correct continuation & 1 & 0.9 \\
\hline
\end{tabular}

of Paradiso-Hardy et al. in Canada [24] and Lallemand et al. in France [10], who showed the timing of administration was correct in $72 \%$ and $61.4 \%$ of cases respectively. Appropriate timing was much higher in the study performed in Jordan (99.1\%) [8], and lower in studies performed in the Netherlands (50\%) [11] and Nicaragua (22\%) [6]. Large-scale observational studies have shown that the rate of surgical site infections decreased by more than 50\% in patients given appropriately timed antibiotic prophylaxis [25-28]. In addition, appropriate antibiotic timing can decrease overall hospitalization costs [29]. The relatively high rate of correct timing of antibiotic administration may be explained by the fact that any time within 0-60 minutes before skin incision is considered appropriate for all types of surgeries according to ASHP guidelines. Therefore, this parameter is less prone to error compared with other parameters, such as antibiotic choice, dose and duration of administration.

In this study non-adherence to practice guidelines resulted in almost US\$ 10 extra cost per patient or US\$ 1527 extra cost for the 15-day period of the study, due to over-use of antibiotics. Some patients were kept in hospital to administer intravenous antibiotics as continuation of prophylactic therapy that was not actually necessary. It should be noted that only the extra cost of the drug itself was calculated; costs would have been much higher if extra days of hospitalization and medical equipment needed to administer intravenous antibiotics were also taken into account.

To put these extra costs into a socioeconomic perspective, the minimum salary of a worker in Shiraz at the time of the study was US\$ 400 per month. Al-Ghamdi et al. in Saudi Arabia reported a minimum government cost of US\$ 565603 annually for misuse of antibiotics [13]. According to surveys performed in Belgium [30] and Turkey [31], adherence to guidelines decreased extra costs due to inappropriate use of antibiotic prophylaxis. In another study it was shown that reducing 24-hour prophylaxis to a single dose, reduces costs without increasing infection rates and results in a potential monthly saving of US\$2 000 [2].

We were not able to collect all data prospectively due to the fact that there was no clinical pharmacist in charge in the surgical ward. Although prospective data collection is more accurate because some information may not be found in patients' charts, we were able to extract all the information we needed from the charts. Care was taken to read all the notes and laboratory reports and make sure that the patient did not have any signs or symptoms of a true infection. Patients with these signs or symptoms were excluded from the study as the antibiotic administration would then be for therapeutic treatment rather than prophylactic purposes.

Although ASHP recommendations were used as rational and evidencedbased international guidelines, ASHP recommendations may not in fact be practical in our patients and setting or for the situation in the Islamic Republic of Iran. This is because different countries and institutions may have different microbial flora, resistance patterns, antibiotic availability, rate of post-operative surgical site infection, and operation room sterility. It is sound practice therefore for each centre to have its own guidelines, although the difference between the guidelines would not be expected to be significant.

Thus developing a local hospital guideline may be more appropriate. Our study shows that there is an urgent need to develop such guidelines for surgical prophylaxis in our hospital. The guidelines should include type of surgery, the optimal time of antibiotic administration, choice of antibiotic and an alternative, address intra-operative re-dosing and duration of use. They should also be based on hospital-specific bacterial epidemiology patterns, the best evidence derived from the literature. The support and collaboration of hospital administrators and medical staff of such guidelines is essential for their development, implementation and maintenance.

\section{References}

1. Garey KW et al. Timing of vancomycin prophylaxis for cardiac surgery patients and the risk of surgical site infections. Journal of Antimicrobial Chemotherapy, 2006, 58:645-650.

2. Fonseca $\mathrm{SN}$ et al. Implementing 1-dose antibiotic prophylaxis for prevention of surgical site infection. Archives of Surgery (Chicago, Ill.), 2006, 141:1109-1113, discussion 1114.
3. Allerberger $\mathrm{F}$ et al. Optimization of antibiotic use in hospitalsantimicrobial stewardship and the EU project ABS international. Chemotherapy, 2008, 54:260-267.

4. Best Practices for Hospital and Health-System Pharmacy. Position and guidance documents of ASHP. Bethesda, MD, American Society of Health-System Pharmacists, 2005-2006. 
5. Wasey N, Baughan J, de Gara CJ. Prophylaxis in elective colorectal surgery: the cost of ignoring the evidence. Canadian Journal of Surgery, 2003, 46:279-284.

6. van Disseldorp J et al. Application of guidelines on preoperative antibiotic prophylaxis in León, Nicaragua. Netherlands Journal of Medicine, 2006, 64:411-416.

7. Askarian M et al. Adherence to American Society of HealthSystem Pharmacists surgical antibiotic prophylaxis guidelines in Iran. Infection Control and Hospital Epidemiology, 2006 , 27:876-878

8. Al-Momany $\mathrm{NH}$ et al. Adherence to international antimicrobial prophylaxis guidelines in cardiac surgery: a Jordanian study demonstrates need for quality improvement. Journal of Managed Care Pharmacy, 2009, 15:262-271.

9. Gorecki $\mathrm{P}$ et al. Antibiotic administration in patients undergoing common surgical procedures in a community teaching hospital: the chaos continues. World Journal of Surgery, 1999, 23:429-432, discussion 433.

10. Lallemand $\mathrm{S}$ et al. Non-observance of guidelines for surgical antimicrobial prophylaxis and surgical-site infections. Pharmacy World \& Science, 2002, 24:95-99.

11. van Kasteren ME et al. Adherence to local hospital guidelines for surgical antimicrobial prophylaxis: a multicentre audit in Dutch hospitals. Journal of Antimicrobial Chemotherapy, 2003, 51:1389-1396.

12. Voit SB et al. Electronic surveillance system for monitoring surgical antimicrobial prophylaxis. Pediatrics, 2005, 116:1317-1322.

13. Al-Ghamdi S, Gedebou M, Bilal NE. Nosocomial infections and misuse of antibiotics in a provincial community hospital, Saudi Arabia. Journal of Hospital Infection, 2002, 50:115-121.

14. Hu S, Liu X, Peng Y. Assessment of antibiotic prescription in hospitalised patients at a Chinese university hospital. Journal of Infection, 2003, 46:161-163.

15. Vaisbrud $V$ et al. Surveillance of antimicrobial prophylaxis for surgical procedures. Infection Control and Hospital Epidemiology, 1999, 20:610-613.

16. Heineck I, Ferreira MB, Schenkel EP. Prescribing practice for antibiotic prophylaxis for 3 commonly performed surgeries in a teaching hospital in Brazil. American Journal of Infection Control, 1999, 27:296-300.

17. Thomas $\mathrm{M}$ et al. Monitoring of antibiotic use in a primary and tertiary care hospital. Journal of Clinical Epidemiology, 1996, 49:251-254.

18. Silver A et al.; The Antibiotic Prophylaxis Study Group. Timeliness and use of antibiotic prophylaxis in selected inpa- tient surgical procedures. American Journal of Surgery, 1996, 171:548-552.

19. Martin C, Pourriat JL. Quality of perioperative antibiotic administration by French anaesthetists. Journal of Hospital Infection, 1998, 40:47-53.

20. Antimicrobial prophylaxis for surgical procedures. In: KodaKimble MA et al., eds. Applied Therapeutics: The clinical use of drugs, 9th ed. Philadelphia, PA, Lippincott Williams \& Wilkins, 2009.

21. Principles of infectious diseases. In: Koda-Kimble MA et al., eds. Applied Therapeutics: The clinical use ofdrugs, 9th ed. Philadelphia, PA, Lippincott Williams \& Wilkins, 2009.

22. Hosoglu $S$ et al. Audit of quality of perioperative antimicrobial prophylaxis. Pharmacy World \& Science, 2009, 31:14-17.

23. Harbarth $\mathrm{S}$ et al. Prolonged antibiotic prophylaxis after cardiovascular surgery and its effect on surgical site infections and antimicrobial resistance. Circulation, 2000, 101:2916-2921.

24. Paradiso-Hardy FL et al. A national survey of antimicrobial prophylaxis in adult cardiac surgery across Canada. Canadian Journal of Infectious Diseases \& Medical Microbiology, 2002, 13:21-27.

25. Burke JP. Maximizing appropriate antibiotic prophylaxis for surgical patients: an update from LDS Hospital, Salt Lake City. Clinical Infectious Diseases, 2001, 33 Suppl 2;S78-S83.

26. Carlès $M$ et al. Improvement of surgical antibiotic prophylaxis: a prospective evaluation of personalized antibiotic kits. Journal of Hospital Infection, 2006, 62:372-375

27. Gyssens IC et al. Optimizing the timing of antimicrobial prophylaxis in surgery: an intervention study. Journal of Antimicrobial Chemotherapy, 1996, 38:301-308.

28. Van Kasteren ME et al. Quality improvement of surgical prophylaxis in Dutch hospitals: evaluation of a multi-site intervention by time series analysis. Journal of Antimicrobial Chemotherapy, 2005, 56:1094-1102.

29. Garey KW et al. Economic benefit of appropriate timing of vancomycin prophylaxis in patients undergoing cardiovascular surgery. Pharmacotherapy, 2008, 28:699-706.

30. Sasse A et al. Surgical prophylaxis in Belgian hospitals: estimate of costs and potential savings. Journal of Antimicrobial Chemotherapy, 1998, 41:267-272.

31. Yalcin AN et al. Increased costs due to inappropriate surgical antibiotic prophylaxis in a university hospital. Journal of Hospital Infection, 2002, 52:228-229. 